\title{
Memory Functioning in Children with Epilepsy: Frontal Lobe Epilepsy, Childhood Absence Epilepsy, and Benign Epilepsy with Centrotemporal Spikes
}

\author{
Ana Filipa Lopes, ${ }^{1,2}$ José Paulo Monteiro, ${ }^{2}$ Maria José Fonseca, \\ Conceição Robalo, ${ }^{3}$ and Mário Rodrigues Simões ${ }^{1}$ \\ ${ }^{1}$ Faculty of Psychology, University of Coimbra, Apartado 6153, 3001-802 Coimbra, Portugal \\ ${ }^{2}$ Neuropaediatric Unit, Garcia de Orta Hospital, Almada, Portugal \\ ${ }^{3}$ Neuropaediatric Unit, Coimbra Paediatric Hospital, Coimbra, Portugal \\ Correspondence should be addressed to Ana Filipa Lopes; anafilipalopes@fpce.uc.pt
}

Received 8 February 2014; Accepted 12 June 2014; Published 3 August 2014

Academic Editor: Cristiano Termine

Copyright ( 2014 Ana Filipa Lopes et al. This is an open access article distributed under the Creative Commons Attribution License, which permits unrestricted use, distribution, and reproduction in any medium, provided the original work is properly cited.

\begin{abstract}
Specific cognitive deficits have been identified in children with epilepsy irrespective of results on intelligence tests. Memory deficits are traditionally attributed to temporal lobe epilepsy, whereas the impact of frontal lobe epilepsy on memory functions has remained controversial. The aim of this study was the examination of memory abilities in other childhood common epilepsy syndromes (frontal lobe epilepsy (FLE), childhood absence epilepsy (CAE), and benign epilepsy with centrotemporal spikes (BECTS)) and the influence of epilepsy-related variables. Memory was examined in 90 children with epilepsy (each epilepsy group consisted of 30 children), aged 6-15, and compared with 30 control children. Children with FLE showed significant deficits in verbal and visual memory. In addition, type of epilepsy, earlier age at epilepsy onset, and longer active duration of epilepsy were associated with memory problems. Seizure frequency and treatment, however, did not influence memory performance. This study indicates that children with FLE show greater risk of developing memory deficits than children with CAE or BECTS, thus highlighting the importance of assessing also memory functions in frontal lobe epilepsy.
\end{abstract}

\section{Introduction}

Memory plays an important role in school performance, as school age children have to learn and integrate new information every day. Memory problems are common in patients with epilepsy and may be differently affected by the cause, course, or treatment of epilepsy. Memory deficits have been documented especially in children with temporal lobe epilepsy [1-7]. But several studies have shown that memory problems may be present in other epilepsy syndromes, such as frontal lobe epilepsy [7-12] and idiopathic generalized epilepsy including childhood absence epilepsy $[7,13,14]$ and benign epilepsy with centrotemporal spikes [15-17].

The study of memory combines the characterization of the phases of encoding, storage, and retrieval of information, which has been associated with temporal as well as frontal networks. Whereas children with temporal lobe epilepsy seem to reveal most of all difficulties with recalling, those with frontal lobe epilepsy seem to be more prone to interference and show difficulties organizing the material to be learned and finding mnemonic strategies $[9,10,18]$. Children with frontal lobe epilepsy experience memory difficulties that have been attributed to primary attention problems and difficulties in applying successful encoding strategies. However this association between memory deficits and frontal lobe epilepsy is still controversial, as some authors have reported no memory difficulties $[19,20]$.

Although memory deficits are more often present in focal epilepsies, some studies have also found memory problems-especially in visual memory-in children with childhood absence epilepsy $[10,13,14,21]$, while others have 
not found significant differences in memory functioning in these epileptic syndromes $[22,23]$. However these children are usually integrated in a miscellanea group that includes not only children with childhood absence epilepsy, but also juvenile absence seizures, juvenile myoclonic epilepsy, and grand mal seizures on awakening, integrating several generalized idiopathic epileptic syndromes.

There is also an extensive literature on the neuropsychological functioning of children and adolescents with benign epilepsy with centrotemporal spikes that includes the study of memory functions. Some studies with this population have identified deficits on verbal memory tasks [16, 24, 25]. Other studies found no memory deficits in this subgroup [22, 26]. In this group of children with epilepsy, memory problems seem secondary to problems on the language domain, as children with benign epilepsy with centrotemporal spikes show deficits that involve most of all verbal functioning [17, 24, 26].

Besides type of epilepsy there are multiple factors that may contribute to memory problems in children with epilepsy. These include age at epilepsy onset [7, 18, 21, 27], duration of active epilepsy [7, 18], seizure frequency [21, 28, $29]$, and treatment [30, 31]. However, only few studies in the paediatric population have studied the impact of these epilepsy-related variables on memory. Moreover, often the evaluation protocols do not include measures of memory.

The aim of this study was to examine memory abilities in children with common childhood epilepsy syndromes (frontal lobe epilepsy (FLE), childhood absence epilepsy (CAE), and benign epilepsy with centrotemporal spikes (BECTS)) and compare them with matched controls. We hypothesize that children with FLE will present worse performances on memory tasks, especially on verbal memory. Finally, we will examine the influence of epilepsy-related variables (type of epilepsy, age at epilepsy onset, duration of active epilepsy, frequency of seizures, and treatment) on memory functions.

\section{Material and Methods}

2.1. Participants. Memory was examined in 90 children with epilepsy (30 with frontal lobe epilepsy (FLE), 30 with childhood absence epilepsy (CAE), 30 with benign epilepsy with centrotemporal spikes (BECTS)), and 30 controls. Children with epilepsy were recruited from neuropaediatric units of the Hospital Garcia de Orta and Coimbra's Paediatric Hospital. This study was approved by the institutional review boards of both institutions and families and children gave their consent to participate. The healthy control children were chosen from the group that was previously used to standardize Coimbra's Neuropsychological Assessment Battery, to match the experimental group for socioeconomic level, age, and gender.

The participants with epilepsy were classified by child neurologists using the International League against Epilepsy criteria [32, 33]. All the children with FLE had performed neuroimaging testing. Children with epilepsy were selected based on the following inclusionary criteria: (i) they were between 6 and 15 years of age; (ii) they were diagnosed with FLE, CAE, or BECTS; (iii) they were administered the Wechsler Intelligence Scale for Children, third edition [34], and obtained a full scale IQ $\geq 70$ [35]; and (iv) they were receiving no more than two antiepileptic medications.

2.2. Measures. Memory functioning was evaluated using memory tests from Coimbra's Neuropsychological Assessment Battery [36]. Coimbra's Neuropsychological Assessment Battery is a comprehensive assessment instrument, directed towards the assessment of Portuguese children's neuropsychological development and functioning. It contains a diversified group of subtests which address the domains of attention, executive functions, memory, language, and motor functions [37]. The different subtests are transformed in scaled scores with a mean of 10 and a standard deviation of 3. The following memory tests were administered.

2.2.1. List Learning. This test assesses the child's ability to learn and evoke a list of words. Children must learn a list of 15 words over four consecutive learning trials (learning). After learning an interference list that is repeated only once, the child is asked to recall the first list of words (immediate recall trial). After an interval of 20 to 30 minutes, the child must recall once more the learning list (delayed recall trial). Finally, 45 words are presented and the child must identify which words belong to the learning list (recognition trial).

2.2.2. Rey Complex Figure Test. This is a classic measure of visual memory, visuospatial constructional ability, and planning. Children are instructed to copy a complex geometric figure and then reproduce it from memory after three minutes (immediate recall) and then 30 minutes (delayed recall). Given the fact that this paper aims to study memory functioning, the copy results will not be described.

2.2.3. Corsi Block Tapping Test. This is a test of visual working memory. Children have to remember blocks that were tapped in sequence by the examiner. Immediately after children repeat the sequence demonstrated by the examiner, these sequences are presented on a wooden board with 9 blocks numbered on the side of the examiner (children do not have visual access to the blocks numeration). At each difficulty level (starting at two blocks) two different trials are presented.

2.3. Statistical Analysis. Statistical analysis was performed using the Statistical Package for the Social Sciences (SPSS, Chicago, IL, USA-version 17.0). Associations between categorical variables were analysed using chi-square test. To test mean differences in demographic and clinical variables and in memory scores across the three types of epilepsy (FLE, CAE, and BECTS), analysis of variance (ANOVA) was used with post hoc analysis using Tukey HSD. Simple regression analysis was used to analyse the effects of epilepsy-related clinical variables (type of epilepsy, age at epilepsy onset, active duration, frequency of seizures, and treatment). Results were judged statistically significant if the $P$ value was identical to or smaller than 0.05 . 


\section{Results}

Table 1 summarizes the demographic (age at testing, gender, and parental education) and clinical characteristics (age at epilepsy onset, seizure frequency, active duration of epilepsy, and treatment) of the participants, 90 children and adolescents with epilepsy (30 FLE; $30 \mathrm{CAE} ; 30 \mathrm{BECTS}$ ), and 30 controls, between the ages of 6 and 15 years old. No significant differences were found between the clinical groups and the control group for age at testing and parental education. However for the variable gender the group with FLE differed from the CAE, BECTS, and control groups. These can be explained by the fact that FLE is more frequent in male children $[8,9]$. We tested for gender differences on the memory tests performed and no differences were found between boys and girls. On the neurological characteristics of the experimental samples no significant differences were observed between the groups for any of the epilepsy-related variables in analysis (age at epilepsy onset, seizure frequency, active duration of epilepsy, and treatment). The group with FLE was composed of 7 children with structural aetiology and 23 with unknown aetiology.

3.1. Differences between Groups. As can be seen in Table 2, significant differences were observed for the following list learning test trials: learning $(F(3,116)=5.039, P=0.003)$, immediate recall $(F(3,116)=3.322, P=0.022)$, and recognition $(F(3,116)=5.191, P=0.002)$ and also for the Corsi block tapping test $(F(3,116)=3.098, P=$ 0.030 ). Post hoc analysis revealed a consistent pattern in which children with FLE showed the worst performance on memory testing: compared to controls, FLE performed worse on the list learning-learning trial $(P=0.001)$; on the list learning-immediate recall trial $(P=0.023)$; on the list learning-recognition trial $(P=0.005)$; and on the Corsi block tapping test $(P=0.017)$. The group with BECTS scored significantly lower than controls on the recognition trial of the list learning task $(P=0.004)$. For the Rey complex figure test (immediate and delayed recall trials) the differences between the clinical groups and the control group did not reach statistical significance.

Given the fact that 7 children from the FLE group had structural lesions, a second analysis including only the other 23 cases with unknown cause was performed. Significant differences were still found for list learning trials-learning $(F(3,109)=5.770, P=0.001)$, immediate recall $(F(3,109)=$ 4.434, $P=0.006)$, and recognition $(F(3,109)=6.779, P=$ $0.000)$-and for the Corsi Block Tapping Test $(F(3,109)=$ 3.057, $P=0.031$ ). Post hoc analysis revealed that children with FLE performed worse than controls on the same 4 trials: on the list learning-learning trial $(P=0.001)$; on the list learning-immediate recall trial $(P=0.005)$; on the list learning-recognition trial $(P=0.000)$; and on the Corsi block tapping test $(P=0.017)$. Also on learning trial of the list learning test the group with FLE had significantly lower scores than CAE $(P=0.047)$ and BECTS $(P=0.017)$.

3.2. Risk Factors Related to Epilepsy. Table 3 shows the results for the linear regression analysis. Regression coefficients were not significant for frequency of seizures and treatment. Lower scores on the list learning test-learning trial (FLE versus AE: $P=0.042$; FLE versus BECTS: $P=0.021$ ) were associated with the type of epilepsy. Also lower scores on the list learning test-learning trial $(P=0.055)$ were related to a longer duration of epilepsy. Finally, lower scores on the Corsi block tapping test $(P=0.002)$ were associated with younger age at epilepsy onset.

\section{Discussion}

Mnemonic deficits are traditionally considered characteristic of temporal lobe epilepsy. However, the presence of memory problems in children with FLE is a question still open in the literature. Results of the present study confirm that children with FLE demonstrate a higher risk of developing memory problems than children with CAE or with BECTS, highlighting the importance of assessing memory functions especially in FLE. Age at epilepsy onset and duration of active epilepsy were the other epilepsy-related variables that were associated with lower memory scores.

4.1. Type of Epilepsy. Our study indicates that children with FLE show greater risk of developing memory problems. Memory deficits occurred mainly during the learning phase of the verbal learning task (list learning test) and the impairment on the immediate recall and recognition trials seems to be the consequence of problems during the initial encoding of information. Verche et al. [38] identified similar verbal memory deficits using the same task, where children with FLE applied encoding and recall strategies less effectively than those of control children. The authors suggest that the deficits found could be explained by the executive functioning deficits commonly detected in patients with FLE. These results appear to be consistent with those of Jambaqué et al. [10] and Lendt et al. [11], who both demonstrated memory impairment did not only occur in participants with temporal lobe epilepsy, but also in children suffering from FLE. These FLE samples showed particularly poor performances in verbal memory tasks that required planning and organizational strategies. Visual memory has been less well studied in children with FLE. Contrary to previous studies [9], we did not find deficits on visual memory, as assessed by the recall trials of Rey complex figure test. On the visual working memory test (Corsi block tapping test) results of children with FLE were lower than the control group, which suggests visuospatial working memory problems. The frontal lobes play an important role on the performance of these tasks, as several studies have demonstrated the activation of the prefrontal cortex for the performance of working memory tasks $[39,40]$. Regarding aetiology of epilepsy, our FLE sample is mainly constituted by an unknown aetiology (77\%). And in fact, authors argue that children with FLE rarely show macrostructural brain abnormalities [8]. The recent work of Braakman et al. $[8,41]$ hypothesized that neuronal injury associated with epilepsy could be expressed as a microstructural or functional abnormality, that simultaneously results 
TABLE 1: Demographic and neurological features.

\begin{tabular}{|c|c|c|c|c|c|}
\hline & $\operatorname{FLE}(N=30)$ & $\operatorname{CAE}(N=30)$ & $\operatorname{BECTS}(N=30)$ & Control $(N=30)$ & $P$ value \\
\hline Age & $\mathrm{M}=10.13(\mathrm{SD}=2.73)$ & $\mathrm{M}=9.93(\mathrm{SD}=2.54)$ & $\mathrm{M}=9.77(\mathrm{SD}=2.43)$ & $\mathrm{M}=10.13(\mathrm{SD}=2.73)$ & 0.937 \\
\hline \multicolumn{6}{|l|}{ Gender } \\
\hline Boys & $77 \%(N=23)$ & $30 \%(N=9)$ & $33 \%(N=10)$ & $50 \%(N=15)$ & \\
\hline Girls & $23 \%(N=7)^{*}$ & $70 \%(N=21)$ & $67 \%(N=20)$ & $50 \%(N=15)$ & 0.001 \\
\hline \multicolumn{6}{|l|}{ Years of education (mother) } \\
\hline Up to 9 th grade & $17 \%(N=5)$ & $23 \%(N=7)$ & $20 \%(N=6)$ & $14 \%(N=4)$ & \\
\hline 9th grade & $30 \%(N=9)$ & $30 \%(N=9)$ & $47 \%(N=14)$ & $30 \%(N=9)$ & \\
\hline 12 th grade & $30 \%(N=9)$ & $20 \%(N=6)$ & $20 \%(N=6)$ & $33 \%(N=10)$ & \\
\hline Superior & $23 \%(N=7)$ & $27 \%(N=8)$ & $13 \%(N=4)$ & $23 \%(N=7)$ & 0.736 \\
\hline Age at onset (years) & $\mathrm{M}=6.40(\mathrm{SD}=3.10)$ & $\mathrm{M}=6.83(\mathrm{SD}=2.32)$ & $\mathrm{M}=6.77(\mathrm{SD}=2.43)$ & & 0.792 \\
\hline \multicolumn{6}{|l|}{ Seizure frequency } \\
\hline No seizures (last 6 months) & $57 \%(N=17)$ & $70 \%(N=21)$ & $60 \%(N=18)$ & & \\
\hline$<1$ a month & $30 \%(N=9)$ & $13 \%(N=4)$ & $37 \%(N=11)$ & & 0.177 \\
\hline$\geq 1$ a month & $13 \%(N=4)$ & $17 \%(N=5)$ & $3 \%(N=1)$ & & \\
\hline Active duration (months) & $M=27.57(S D=36.24)$ & $\mathrm{M}=22.63(\mathrm{SD}=17.95)$ & $M=20.90(S D=26.44)$ & & 0.632 \\
\hline \multicolumn{6}{|l|}{ Treatment } \\
\hline No medication & $7 \%(N=2)$ & $13 \%(N=4)$ & $27 \%(N=8)$ & & \\
\hline Monotherapy & $80 \%(N=24)$ & $73 \%(N=22)$ & $73 \%(N=22)$ & & 0.087 \\
\hline Duotherapy & $13 \%(N=4)$ & $13 \%(N=4)$ & - & & \\
\hline
\end{tabular}

${ }^{*}$ Different from Control $(P=0.032)$, from CAE $(P=0.000)$, and from BECTS $(P=0.001)$.

TABLE 2: Memory tests scores.

\begin{tabular}{|c|c|c|c|c|c|c|c|}
\hline & $\begin{array}{c}\operatorname{FLE}(N=30) \\
\text { Mean }(\mathrm{SD})\end{array}$ & $\begin{array}{c}\operatorname{CAE}(N=30) \\
\text { Mean }(\mathrm{SD})\end{array}$ & $\begin{array}{c}\text { BECTS }(N=30) \\
\text { Mean }(\mathrm{SD})\end{array}$ & $\begin{array}{c}\text { Control }(N=30) \\
\text { Mean }(\mathrm{SD})\end{array}$ & $F$ & df & $\begin{array}{c}P \text { value } \\
\text { (ANOVA) }\end{array}$ \\
\hline \multicolumn{8}{|l|}{ LIST learning } \\
\hline Learning & $6.33(3.45)^{* * *}$ & $8.13(3.28)$ & $8.47(2.90)$ & $9.39(2.82)$ & 5.039 & 3,116 & 0.003 \\
\hline Immediate recall & $7.17(3.06)^{*}$ & $8.70(3.38)$ & $7.73(3.08)$ & $9.38(2.20)$ & 3.322 & 3,116 & 0.022 \\
\hline Delayed recall & $7.83(2.60)$ & $8.90(3.07)$ & $8.40(3.05)$ & $9.39(2.27)$ & 1.744 & 3,116 & 0.162 \\
\hline Recognition & $7.13(3.79)^{* *}$ & $8.20(3.63)$ & $7.07(3.85)^{* *}$ & $10.25(2.72)$ & 5.191 & 3,116 & 0.002 \\
\hline \multicolumn{8}{|l|}{ Rey complex figure test } \\
\hline Immediate recall & $8.33(3.27)$ & $9.00(3.27)$ & $8.87(2.85)$ & $9.57(2.67)$ & 0.757 & 3,116 & 0.521 \\
\hline Delayed recall & $8.50(3.26)$ & $8.63(3.73)$ & $8.47(3.09)$ & $9.59(2.84)$ & 0.810 & 3,116 & 0.491 \\
\hline Corsi block tapping test & $7.40(3.14)^{*}$ & $8.97(3.44)$ & $8.77(3.27)$ & $9.85(2.74)$ & 3.098 & 3,116 & 0.030 \\
\hline
\end{tabular}

${ }^{*}$ Different from control $(P \leq 0.05)$.

${ }^{* *}$ Different from control $(P \leq 0.01)$.

${ }^{* * *}$ Different from control $(P \leq 0.001)$.

in neurobehavioral comorbidities. 32 children with cryptogenic FLE underwent neuropsychological assessment and structural and functional magnetic resonance imaging and decreased functional frontal lobe connectivity was associated with cognitive impairment in FLE.

The existing literature suggests that most children with BECTS show intact memory skills [17, 22, 24, 26]. Our study only identified difficulties in the recalling phase (recognition) of the list learning task, presenting mean normal scores during learning and recall on all of the other memory tasks. Attention problems that have been previously reported in children with BECTS [17, 42-44] may justify the difficulties with recognition of items previously learned and failure to resist interference.
It is very important to consider memory problems in the context of other cognitive pathology, namely, in the domains of attention, executive functions, and language. Considering that memory deficits in children seem to be a part of a much more diffuse impairment $[27,45]$. Clinically, the memory profile of each child should be analysed in the context of other neurocognitive abilities and this is only possible when conducting extensive and comprehensive neuropsychological assessment protocols.

4.2. Age at Epilepsy Onset and Duration of Active Epilepsy. Concerning the impact of the clinical variables related to epilepsy, besides type of epilepsy, age at epilepsy onset and 


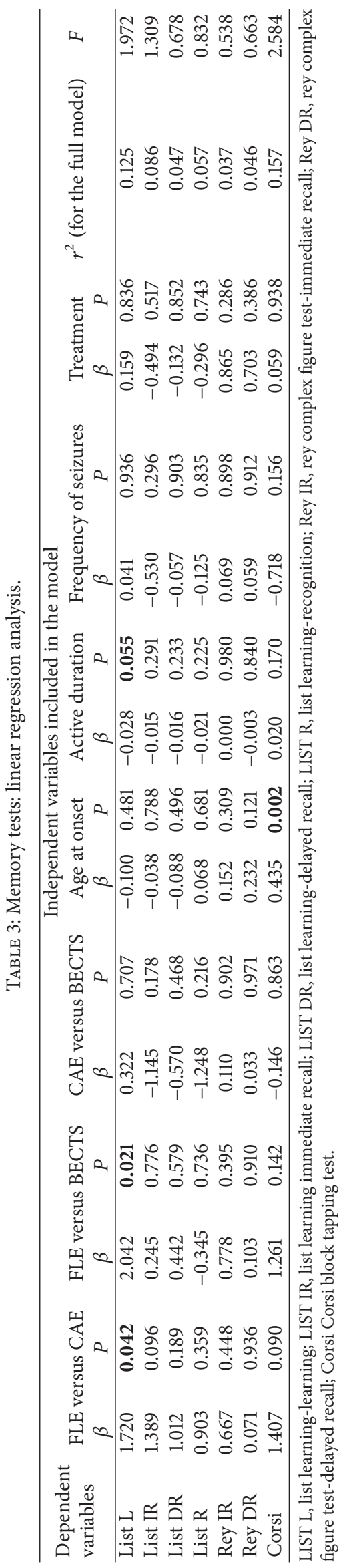


duration of active epilepsy were the most significant risk factors for memory difficulties in our study. Seizure frequency and the number of antiepileptic drugs were not associated with memory functioning.

In the present study younger age at epilepsy onset was associated with poorer performance on the Corsi block tapping test that assesses visual working memory. The negative impact of an earlier age at epilepsy onset on cognitive functions has been largely documented, including memory functions $[12,18,21]$. In our study, an earlier age at epilepsy onset was related to a lower performance on a task that assesses visual working memory but that is also sensitive to attention and executive functions deficits. This is a common finding on other studies. Kernan et al. [21] studied a group of children with CAE and found a significant effect for age at epilepsy onset on a digit span task testing verbal working memory abilities. And Riva et al. [18] documented a significant correlation between age at epilepsy onset of epilepsy and performance on a list learning test; these children had difficulties on learning and using clusters and these impaired their scores on recall tasks. In our study earlier age at epilepsy onset only influenced results on the task that is sensitive to executive functions performance (Corsi block tapping test). Executive functions are a heterogeneous construct that includes several subdomains (such as planning, organization, working memory, inhibition, problem solving, fluency, initiative, and anticipation) that are difficult to dissociate from other constructs, such as memory, attention, or processing speed. Executive functions are subserved by the frontal lobes and these are the latest brain regions to develop, as maturation continues throughout adolescence [46]. For this reason early age at epilepsy onset is a major risk factor for difficulties on executive functions-related skills.

A longer duration of active epilepsy predicted memory difficulties on the learning phase of the list learning task. That is, children and adolescents with more years of active epilepsy were more likely to have problems on the acquisition and consolidation of knowledge, which will probably impact on their school results. A few studies have documented the impact of active duration of epilepsy on memory functions. Schoenfeld et al. [47] investigated a group of children with complex partial seizures and observed an association of a longer duration of active epilepsy with lower scores on visual memory. Nolan's group [7] studied memory functions in three childhood epilepsy syndromes (temporal lobe epilepsy, FLE, and CAE) and found that duration of epilepsy correlated negatively with memory function and thus was the most significant risk factor for memory problems. This was confirmed by another study that included only children with FLE: Riva et al. [18] found that performance on a list learning task (number of correct answers and short and long-term recall) correlated with the duration of the disorder.

4.3. Limitations. One of the limitations of our study was the fact that regarding verbal memory assessment we only included the list learning test, which is considered a representative task to assess memory (verbal memory). List learning is a task that includes repeated presentations of test material and is very susceptible to attention and executive functions problems.

It is frequently reported by parents and teachers that children with epilepsy experience memory deficits impairing their everyday life. Traditional memory tests may not capture all the real memory capabilities of children with epilepsy. Everyday memory might be influenced much more by attention problems than standard memory testing $[48,49]$. Therefore future studies should include measures of everyday memory on neuropsychological assessment protocols, as well as other memory tests (story recall, paired word learning, sentence memory, visual recall, and memory for faces).

\section{Conclusions}

The present study demonstrates that children with FLE show significant deficits in verbal and visual memory. In addition, type of epilepsy, earlier age at epilepsy onset, and longer active duration of epilepsy were associated with memory problems. Our research findings underline the importance of offering early assessments, especially for children with FLE, with a longer duration and an early age at epilepsy onset, with extensive neuropsychological assessment protocols that include several measures of memory. Knowing the outcome of these groups of children with epilepsy in memory tests gives clinicians the possibility to establish adequate and timely school intervention plans to diminish the negative influence that this memory problems might have on their academic achievement.

\section{Conflict of Interests}

None of the authors has any conflict of interests to disclose.

\section{Acknowledgments}

The authors are very grateful to the participating children and their families. They thank Cláudia Lopes and Katrin Schulze for their helpful comments on previous versions of the paper. This study was supported by the Portuguese Foundation for Science and Technology (FCT-Fundação para a Ciência e Tecnologia) (SFRH/BD/40758/2007).

\section{References}

[1] M. Cohen, "Auditory/verbal and visual/spatial memory in children with complex partial epilepsy of temporal lobe origin," Brain and Cognition, vol. 20, no. 2, pp. 315-326, 1992.

[2] F. Cormack, F. Vargha-Khadem, S. J. Wood, J. H. Cross, and T. Baldeweg, "Memory in paediatric temporal lobe epilepsy: effects of lesion type and side," Epilepsy Research, vol. 98, no. 2-3, pp. 255-259, 2012.

[3] L. M. Gonzalez, V. A. Anderson, S. J. Wood, L. A. Mitchell, and A. S. Harvey, "The localization and lateralization of memory deficits in children with temporal lobe epilepsy," Epilepsia, vol. 48, no. 1, pp. 124-132, 2007.

[4] C. A. Guimarães, L. M. Li, P. Rzezak et al., “Temporal lobe epilepsy in childhood: comprehensive neuropsychological 
assessment," Journal of Child Neurology, vol. 22, no. 7, pp. 836840,2007

[5] C. Helmstaedter, "Effects of chronic temporal lobe epilepsy on memory functions," in Cognitive Dysfunction in Children with Temporal Lobe Epilepsy, A. Arzimanoglou, A. Aldenkamp, H. Cross, M. Lassonde, S. Moshé, and Schmitz, Eds., pp. 13-30, John Libbey Eurotext, Paris, France, 2005.

[6] I. Jambaqué, G. Dellatolas, M. Fohlen et al., "Memory functions following surgery for temporal lobe epilepsy in children," Neuropsychologia, vol. 45, no. 12, pp. 2850-2862, 2007.

[7] M. A. Nolan, M. A. Redoblado, S. Lah et al., "Memory function in childhood epilepsy syndromes," Journal of Paediatrics and Child Health, vol. 40, no. 1-2, pp. 20-27, 2004.

[8] H. M. H. Braakman, D. M. Ijff, M. J. Vaessen et al., "Cognitive and behavioural findings in children with frontal lobe epilepsy," European Journal of Paediatric Neurology, vol. 16, no. 6, pp. 707715, 2012.

[9] M. T. Hernandez, H. C. Sauerwein, I. Jambaqué et al., "Attention, memory, and behavioral adjustment in children with frontal lobe epilepsy," Epilepsy and Behavior, vol. 4, no. 5, pp. 522-536, 2003.

[10] I. Jambaqué, G. Dellatolas, O. Dulac, G. Ponsot, and J. Signoret, "Verbal and visual memory impairment in children with epilepsy," Neuropsychologia, vol. 31, no. 12, pp. 1321-1337, 1993.

[11] M. Lendt, U. Gleissner, C. Helmstaedter, R. Sassen, H. Clusmann, and C. E. Elger, "Neuropsychological outcome in children after frontal lobe epilepsy surgery," Epilepsy and Behavior, vol. 3, no. 1, pp. 51-59, 2002.

[12] J. Prévost, A. Lortie, D. Nguyen, M. Lassonde, and L. Carmant, "Nonlesional frontal lobe epilepsy (FLE) of childhood: clinical presentation, response to treatment and comorbidity," Epilepsia, vol. 47, no. 12, pp. 2198-2201, 2006.

[13] Y. Henkin, M. Sadeh, S. Kivity, E. Shabtai, L. Kishon-Rabin, and N. Gadoth, "Cognitive function in idiopathic generalized epilepsy of childhood," Developmental Medicine \& Child Neurology, vol. 47, no. 2, pp. 126-132, 2005.

[14] P. Pavone, R. Bianchini, R. R. Trifiletti, G. Incorpora, A. Pavone, and E. Parano, "Neuropsychological assessment in children with absence epilepsy," Neurology, vol. 56, no. 8, pp. 1047-1051, 2001.

[15] C. Croona, M. Kihlgren, S. Lundberg, O. Eeg-Olofsson, and K. E. Eeg-Olofsson, "Neuropsychological findings in children with benign childhood epilepsy with centrotemporal spikes," Developmental Medicine and Child Neurology, vol. 41, no. 12, pp. 813-818, 1999.

[16] E. Northcott, A. M. Connolly, A. Berroya et al., "The neuropsychological and language profile of children with benign rolandic epilepsy," Epilepsia, vol. 46, no. 6, pp. 924-930, 2005.

[17] F. Pinton, B. Ducot, J. Motte et al., "Cognitive functions in children with benign childhood epilepsy with centrotemporal spikes (BECTS)," Epileptic Disorders, vol. 8, no. 1, pp. 11-23, 2006.

[18] D. Riva, G. Avanzini, S. Franceschetti et al., "Unilateral frontal lobe epilepsy affects executive functions in children," Neurological Sciences, vol. 26, no. 4, pp. 263-270, 2005.

[19] K. Culhane-Shelburne, L. Chapieski, M. Hiscock, and D. Glaze, "Executive functions in children with frontal and temporal lobe epilepsy," Journal of the International Neuropsychological Society, vol. 8, no. 5, pp. 623-632, 2002.

[20] B. Høie, A. Mykletun, P. E. Waaler, H. Skeidsvoll, and K. Sommerfelt, "Executive functions and seizure-related factors in children with epilepsy in western Norway," Developmental Medicine and Child Neurology, vol. 48, no. 6, pp. 519-525, 2006.

[21] C. L. Kernan, R. Asarnow, P. Siddarth et al., "Neurocognitive profiles in children with epilepsy," Epilepsia, vol. 53, no. 12, pp. 2156-2163, 2012.

[22] C. Hommet, C. Billard, J. Motte et al., "Cognitive function in adolescents and young adults in complete remission from benign childhood epilepsy with centro-temporal spikes," Epileptic Disorders, vol. 3, no. 4, pp. 207-215, 2001.

[23] J. Williams, T. Phillips, M. L. Griebel et al., "Patterns of memory performance in children with controlled epilepsy on the CVLTC," Child Neuropsychology, vol. 7, no. 1, pp. 15-20, 2001.

[24] H. Goldberg-Stern, O. M. Gonen, M. Sadeh, S. Kivity, A. Shuper, and D. Inbar, "Neuropsychological aspects of benign childhood epilepsy with centrotemporal spikes," Seizure, vol. 19, no. 1, pp. 12-16, 2010.

[25] C. Vago, S. Bulgheroni, S. Franceschetti, A. Usilla, and D. Riva, "Memory performance on the California Verbal Learning Test of children with benign childhood epilepsy with centrotemporal spikes," Epilepsy and Behavior, vol. 13, no. 4, pp. 600-606, 2008.

[26] S. Völk-Kernstock, S. Bauch-Prater, E. Ponocny-Seliger, and M. Feucht, "Speech and school performance in children with benign partial epilepsy with centro-temporal spikes," Seizure, vol. 18, no. 5, pp. 320-326, 2009.

[27] C. Helmstaedter and M. Lendt, "Neuropsychological outcome of temporal and extratemporal lobe resections in children," in Neuropsychology of Childhood Epilepsy, I. Jambaqué, M. Lassonde, and O. Dulac, Eds., pp. 215-27, Kluwer Academic/Plenum, New York, NY, USA, 2001.

[28] U. Kramer, S. Kipervasser, M. Y. Neufeld, I. Fried, S. Nagar, and F. Andelman, "Is there any correlation between severity of epilepsy and cognitive abilities in patients with temporal lobe epilepsy?" European Journal of Neurology, vol. 13, no. 2, pp. 130 134, 2006.

[29] B. Jocic-Jakubi and N. J. Jovic, "Verbal memory impairment in children with focal epilepsy," Epilepsy and Behavior, vol. 9, no. 3, pp. 432-439, 2006.

[30] A. P. Aldenkamp, "Cognitive side-effects of antiepileptic drugs," in Neuropsychology of Childhood Epilepsy, I. Jambaqué, M. Lassonde, and O. Dulac, Eds., pp. 257-267, Kluwer Academic Publishers/Plenum Publishers, New York, NY, USA, 2001.

[31] P. S. Fastenau, C. S. Johnson, S. M. Perkins, A. W. Byars, T. J. deGrauw, and J. K. Austin, "Neuropsychological status at seizure onset: risk factors for early cognitive deficits," Neurology, vol. 73, pp. 526-534, 2009.

[32] "Commission on classification and terminology of the international league against epilepsy. Proposal for revised classification of epilepsies and epileptic syndromes," Epilepsia, vol. 30, pp. 389-399, 1989.

[33] A. T. Berg, S. F. Berkovic, M. J. Brodie et al., "Revised terminology and concepts for organization of seizures and epilepsies: report of the ILAE Commission on Classification and Terminology, 2005-2009," Epilepsia, vol. 51, no. 4, pp. 676685, 2010.

[34] D. Wechsler, Wechsler Intelligence Scale for Children-Third Edition: Manual, Cegoc, Lisboa, Portugal, 3rd edition, 2003.

[35] A. F. Lopes, M. R. Simões, J. P. Monteiro et al., "Intellectual functioning in children with epilepsy: frontal lobe epilepsy, childhood absence epilepsy and benign epilepsy with centrotemporal spikes," Seizure, vol. 22, no. 10, pp. 886-892, 2013. 
[36] M. R. Simões, C. P. Albuquerque, M. S. Pinho et al., Coimbra's Neuropsychological Assessment Battery, Lisboa, Portugal, Cegoc, In Press.

[37] C. P. Albuquerque and M. R. Simões, "Rapid naming tests: developmental course and relations with neuropsychological measures," The Spanish Journal of Psychology, vol. 13, no. 1, pp. 88-100, 2010.

[38] E. Verche, M. Cairós, and S. Hernández, "Neuropsychological assessment of verbal memory of children and adolescents with frontal lobe epilepsy," Revue Neurologique, vol. 53, p. 627, 2011.

[39] E. E. Smith and J. Jonides, "Working memory: a view from neuroimaging," Cognitive Psychology, vol. 33, no. 1, pp. 5-42, 1997.

[40] A. Baddeley and R. Logie, "Working memory: the multiplecomponent model," in Models of Working Memory, A. Miyake and P. Shah, Eds., pp. 28-61, Cambridge University Press, New York, NY, USA, 1999.

[41] H. M. H. Braakman, M. J. Vaessen, J. F. A. Jansen et al., "Frontal lobe connectivity and cognitive impairment in pediatric frontal lobe epilepsy," Epilepsia, vol. 54, no. 3, pp. 446-454, 2013.

[42] L. Deltour, V. Quaglino, M. Barathon, A. De Broca, and P. Berquin, "Clinical evaluation of attentional processes in children with benign childhood epilepsy with centrotemporal spikes (BCECTS)," Epileptic Disorders, vol. 9, no. 4, pp. 424-431, 2007.

[43] B. Giordani, A. F. Caveney, D. Laughrin et al., "Cognition and behavior in children with benign epilepsy with centrotemporal spikes (BECTS)," Epilepsy Research, vol. 70, no. 1, pp. 89-94, 2006.

[44] P. M. Kavros, T. Clarke, L. J. Strug, J. M. Halperin, N. J. Dorta, and D. K. Pal, "Attention impairment in rolandic epilepsy: systematic review," Epilepsia, vol. 49, no. 9, pp. 1570-1580, 2008.

[45] K. van Rijckevorsel, "Cognitive problems related to epilepsy syndromes, especially malignant epilepsies," Seizure, vol. 15, no. 4, pp. 227-234, 2006.

[46] M. T. Hernandez, H. C. Sauerwein, E. Guise et al., "Neuropsychology of frontal lobe epilepsy in children," in Neuropsychology of Childhood Epilepsy, I. Jambaqué, M. Lassonde, and O. Dulac, Eds., pp. 103-111, Kluwer Academic/Plenum Publishers, New York, NY, USA, 2001.

[47] J. Schoenfeld, M. Seidenberg, A. Woodard et al., "Neuropsychological and behavioral status of children with complex partial seizures," Developmental Medicine and Child Neurology, vol. 41, no. 11, pp. 724-731, 1999.

[48] D. S. Kadis, M. Stollstorff, I. Elliott, L. Lach, and M. L. Smith, "Cognitive and psychological predictors of everyday memory in children with intractable epilepsy," Epilepsy and Behavior, vol. 5, no. 1, pp. 37-43, 2004.

[49] M. L. Smith and E. R. Vriezen, "Everyday memory in children with epilepsy," Epilepsia, vol. 38, article 165, 1997. 


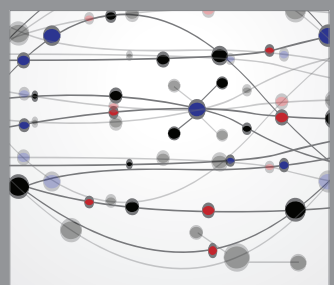

The Scientific World Journal
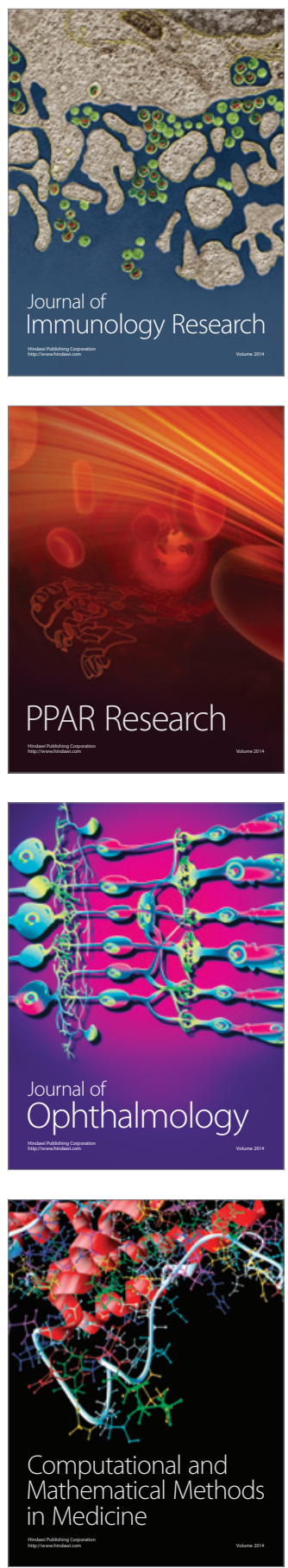

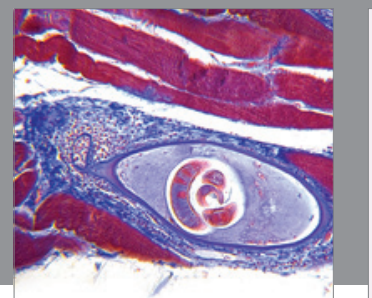

Gastroenterology

Research and Practice
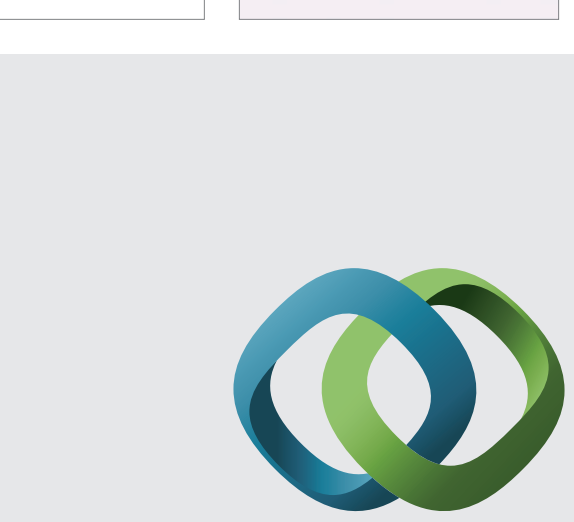

\section{Hindawi}

Submit your manuscripts at

http://www.hindawi.com
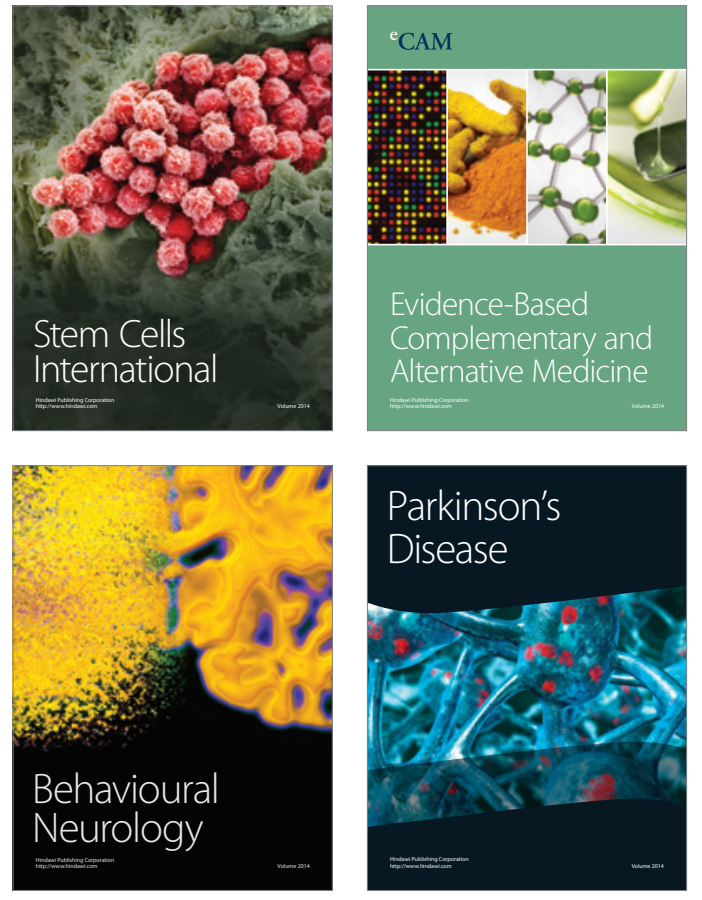
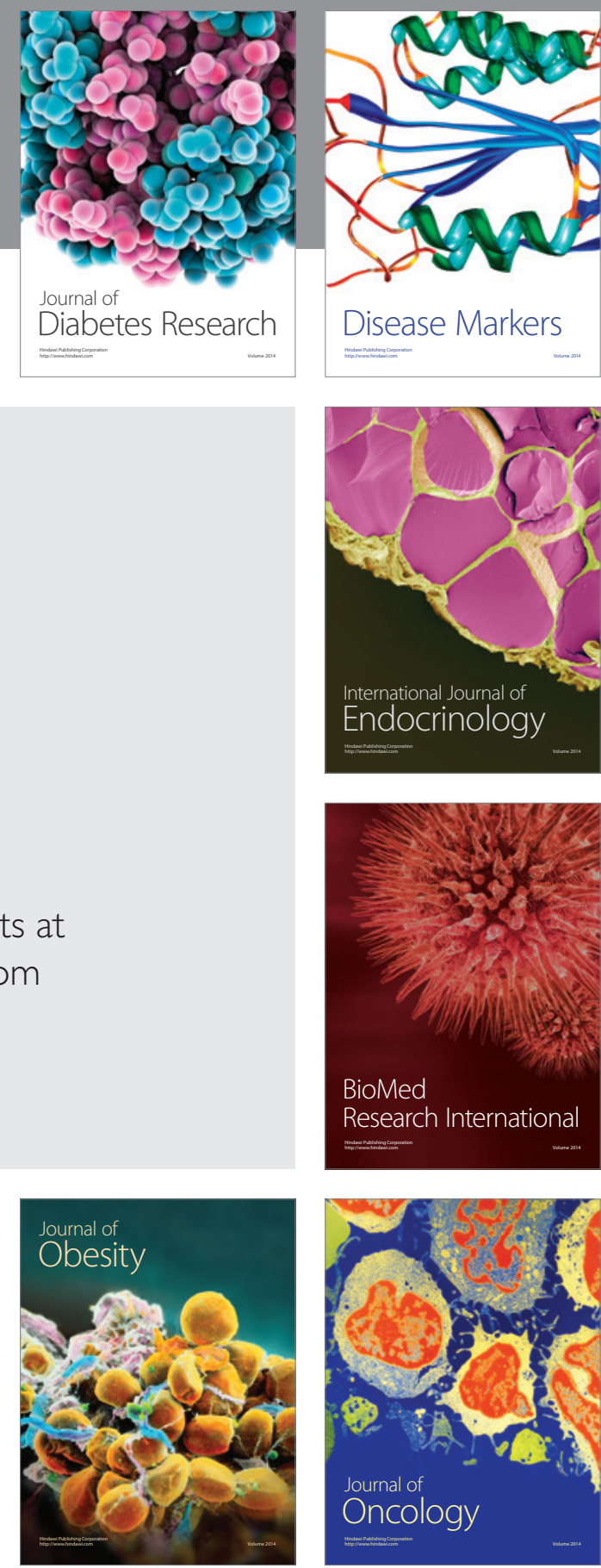

Disease Markers
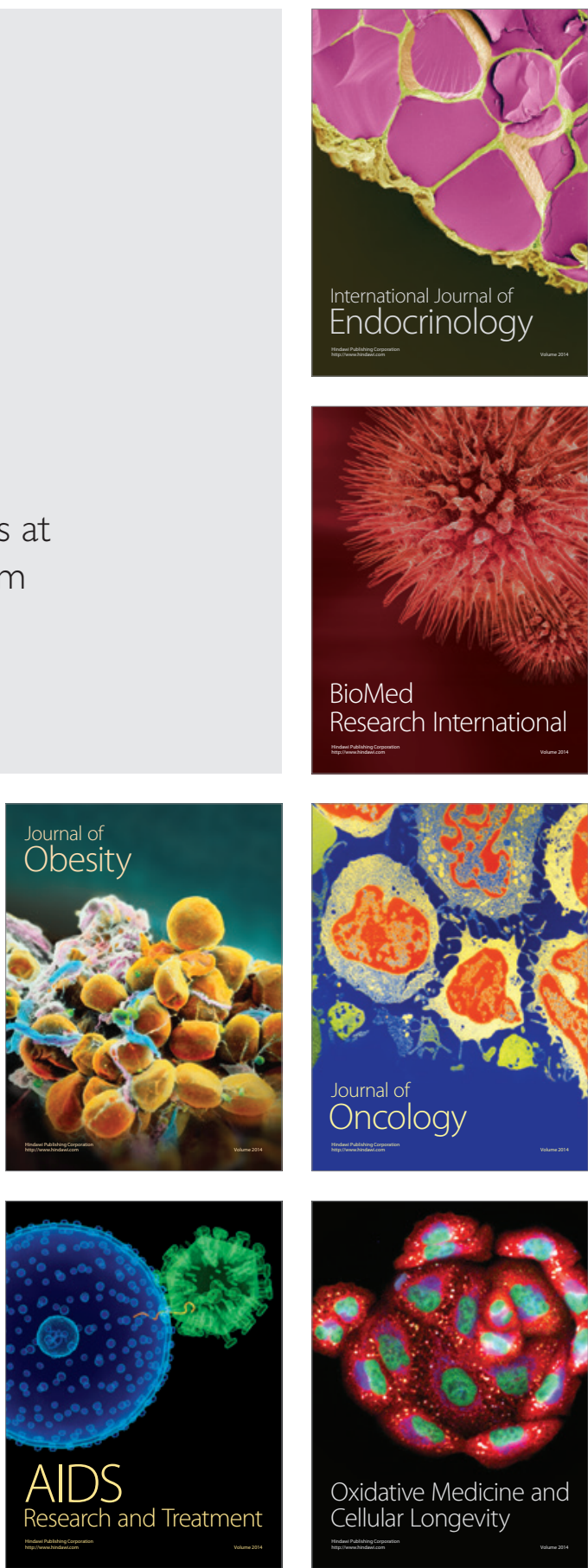